\title{
An investigation of the near-wake flow structure of a cylinder with guiding plates
}

\author{
Fırat Ekinci ${ }^{1}$, Erhan Firat ${ }^{2, *}$, Göktürk M. Özkan ${ }^{3,4}$, and Hüseyin Ak1l11 ${ }^{4}$ \\ ${ }^{1}$ Adana Science and Technology University, Department of Energy System Engineering, 01180, Adana, Turkey \\ ${ }^{2}$ Munzur University, Department of Mechanical Engineering, 62000, Tunceli, Turkey \\ ${ }^{3}$ Delft University of Technology, Process and Energy Department, 2628 CA, Delft, Netherlands \\ ${ }^{4}$ Çukurova University, Department of Mechanical Engineering, 01330, Adana, Turkey
}

\begin{abstract}
In this study, the flow behind a circular cylinder with a pair of outer identical guiding plates was investigated using particle image velocimetry (PIV) for various angular positions of the plates (i.e. $\alpha= \pm 70^{\circ}$, $\pm 100^{\circ}$, and $\pm 130^{\circ}$ ). The gaps between these plates and cylinder are equal and are $0.3 D$. Experiments were carried out at a subcritical Reynolds $\left(R e=\rho \cdot U_{\infty} \cdot D / \mu\right)$ number of 7500, based on the cylinder diameter $(D)$ and the flow velocity $\left(U_{\infty}\right)$. The features of the near-wake with and without the guiding plates were interpreted in terms of patterns of time-averaged vorticity and streamlines, time-averaged and fluctuating velocity components. The spectral analysis was also carried out to determine the time-dependent variation of the transverse velocity at given locations in the near-wake. Two-dimensional computations of flow around circular cylinders with and without guiding plates have also been performed to predict the timeaveraged and root-mean-square of force coefficients of the various models. It was seen that the guiding plates at an appropriate angular position can lead to substantial attenuation, or retardation, of the process of large-scale vortex formation in the near-wake, thus can lead to vortex-induced vibration (VIV) suppression without any increase in drag.
\end{abstract}

\section{Introduction}

When two-dimensional bodies that are not streamlined with respect to the free-stream direction (high-rise structural systems, bridge piers, some parts of onshore/ offshore systems, etc) subjected to a fluid flow at adequately large Reynolds numbers $(R e)$, each of the shear layers from this body roll up in an alternating, periodic fashion, into large-scale vortices (Kármán vortices) behind these bodies (near-wake). Relatively high aerodynamic/hydrodynamic drag and fluctuating forces on the body are several natural consequences of the Kármán vortex formation. These forces are generally undesired. To decrease them on the body, shear layers must be controlled before (boundary layer) and/or after (free shear layers) the separation [1-10]. The aim of this study is firstly to alter the near-wake structure of the cylinder using guiding plates, and then, to interpret the foregoing physics of the near-wake in terms of patterns of time-averaged vorticity and streamlines, timeaveraged and fluctuating velocity components, and spectral analysis; secondly to predict the trend of timeaveraged drag coefficient and root-mean-square of the fluctuating lift coefficient numerically in the absence of the direct force measurements.

\section{Experimental setup}

The experiments were carried out at Çukurova University in a closed circuit water channel with a test section of $8000 \mathrm{~mm}$ long, $1000 \mathrm{~mm}$ wide $(W)$ and 750 $\mathrm{mm}$ high. The test section has $15 \mathrm{~mm}$ thick Plexiglas walls to allow optical access. The water level was maintained at $500 \pm 2 \mathrm{~mm}(H)$. The water was pumped
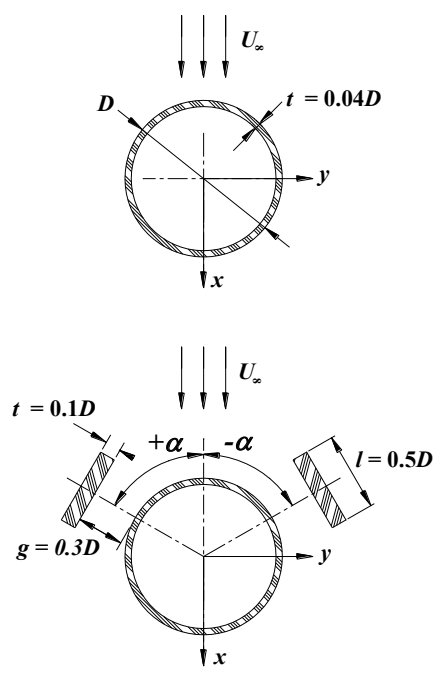

Fig. 1. Cross-sectional view of the circular cylinder without (upper one) and with guiding plates.

\footnotetext{
* Corresponding author: efirat@munzur.edu.tr
} 
from the downstream reservoir through external piping into the upstream reservoir. It then passed through flow straightener, 2:1 contraction and test section of the channel. The water flow was driven by a $15 \mathrm{~kW}$ centrifugal pump. The rotation speed of the pump was controlled by a variable frequency drive controller unit and set to a constant value during tests. A water channel velocity of $0.140 \mathrm{~m} / \mathrm{s}$ gives a Reynolds number of $(R e)$ approximately 7500 , based on the cylinder diameter $(D)$. The free-stream turbulence intensity $\left(T u_{x}\right)$ was less than $1.45 \%$.

One cylinder model without any modification (BC) and three cylinder models with guiding plates at various angular plate positions $\left(\alpha= \pm 70^{\circ}, \pm 100^{\circ}\right.$ and $\left.\pm 130^{\circ}\right)$ were investigated (Fig. 1). The models made of Plexiglas were mounted vertically in the test section of the water channel. Cylinder models were producing a maximum blockage ratio $(B R=D / W)$ of approximately $8.5 \%$ and have an aspect ratio $(A R=l / D)$ of 8 . Guiding plates may undergo large deflections due to the hydrodynamic load. To prevent this, relatively thick plates were employed.

It was stated that a reasonable representation of twodimensional flow can be achieved for models with an aspect ratio greater than about 6 between walls or end plates [11]. Our models fulfill this requirement; therefore, two-dimensional, two-component digital particle image velocimetry (Dantec Dynamics A/S) measurements were obtained only in horizontal plane $(x-$ $y$ ) which is parallel to the floor of the channel test section and $250 \mathrm{~mm}$ higher than it (mid-depth). A double pulsed Nd:YAG laser with a wavelength of $532 \mathrm{~nm}$ (New Wave Research, Solo 120XT) was used to illuminate the above-mentioned plane. The laser sheet was perpendicular to the axis of the cylinder. The flow was seeded by silver-coated hollow glass spheres with a nominal particle diameter of $10 \mu \mathrm{m}$. One CCD camera with a spatial resolution of $1200 \times 1600$ pixels (Dantec Dynamics, FlowSense 2M) was used to detect the movement of seeding particles. The camera was equipped with $60 \mathrm{~mm}$ lens (Nikon AF Micro-Nikkor $60 \mathrm{~mm} \mathrm{f} / 2.8 \mathrm{D}$ ). Each frame covered an area of approximately $158 \times 210.7 \mathrm{~mm}^{2}(3.16 D \times 4.21 D)$. Each vector in an instantaneous vector map was obtained by using frame to frame adaptive correlation technique (double-frame mode). The size of the dimension of interrogation area in pixels was $32 \times 32$. The percentage amount of overlap was 50 . Each vector map contained $7326(74 \times 99)$ instantaneous velocity vectors. It was seen that 1000 instantaneous vector maps can sufficiently represent the time-averaged vector map. Therefore, the image pairs were recorded at $10 \mathrm{~Hz}$ (at least 17 times higher than the vortex shedding frequency of the bare cylinder) for $100 \mathrm{~s}$ to produce 1000 independent vector map samples.

\section{Numerical methods}

It is known that today's CFD codes are generally unable to predict hydrodynamic coefficients with an acceptable degree of reliability but yield reasonable results for many practical engineering problems. Therefore, our aim here is to predict the trend of hydrodynamic statistics (i.e. time-averaged drag coefficient, root-mean-square of the fluctuating lift coefficient) in the absence of the direct force measurements.

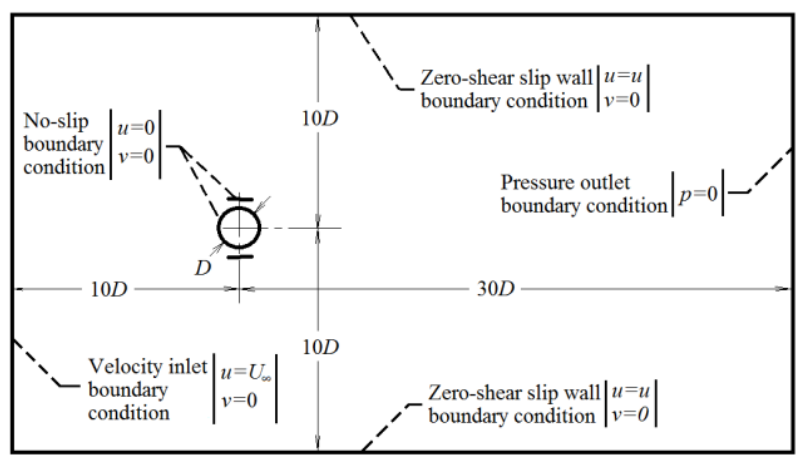

Fig. 2. Geometry of computational domain where length is in diameter of the cylinder $(D)$ and boundary conditions. For the present computations, the inflow boundary condition is taken from the experimental results.

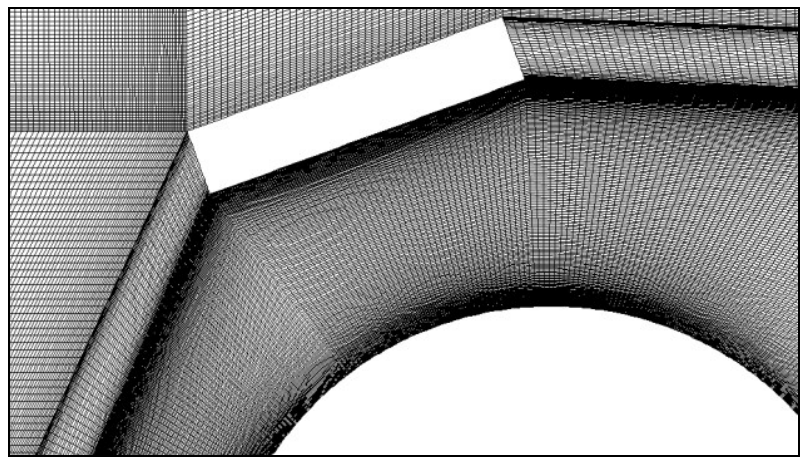

Fig. 3. Image showing near field meshes from the cylinder for A70 case before the mesh adaption process. Flow is from left to right. Computational domain was divided into various blocks with respect to the case, but each block was meshed with structured grid.

Computations were performed using the commercial CFD code ANSYS Fluent 6.3. A Reynolds-averaged Navier-Stokes (RANS) approach was taken due to the lack of the computational effort. The shear stress transport (SST) $k-\omega$ turbulence model which is developed by Menter and provided in FLUENT was chosen since it shows a better performance in complex flows with boundary layer separation when compared to RANS based one- and two-equations turbulence models $[13,14]$. Second-order accuracy is used for the viscous terms. The diffusion terms in the discretized governing equations are central-differenced and second-order accurate. The second-order implicit scheme is adopted for the temporal discretization. SIMPLE (Semi-Implicit Method for the Pressure-Linked Equation) was adopted as pressure-velocity coupling algorithm. The time step was obtained from the quotient of approximate vortex shedding period of the single square prism at $R e=7500$ by $500(\Delta t=0.0034 \mathrm{~s})$ in order to avoid any unphysical oscillation and obtain a smooth time-independent solution. Although the initial mesh around the cylinder and guiding plates are fine, refinements close to the cylinder surface, plate surfaces and in the wake of the cylinder using boundary adaption and velocity gradient 
adaption were also made. The number of cells was varied from 700000 to 1000000 after adaption processes. The height of the first mesh on the cylinder surface was decreased to about $1 \times 10^{-4} \mathrm{D}$ after adaption process. For bare cylinder, the predictions are compared with experimental and numerical results (Table 1). It is clear from the table 1 that the SST $k-\omega$ turbulence model results in over-prediction of hydrodynamic force and vortex shedding values compared with experiment and LES.

Table 1. Comparison of the current results with those of other authors for bare cylinder.

\begin{tabular}{|l|c|c|c|c|}
\hline \multicolumn{1}{|c|}{ Reference/Technique } & $\boldsymbol{R e}$ & $\boldsymbol{C D}_{\boldsymbol{D}}$ & $\boldsymbol{C}_{\boldsymbol{L} \boldsymbol{R} \boldsymbol{S} \boldsymbol{S}}$ & $\boldsymbol{S} \boldsymbol{t}$ \\
\hline$[12]$ / Experimental & 7500 & - & 0.350 & 0.203 \\
\hline$[15]$ / Experimental & 7233 & 1.12 & 0.177 & 0.235 \\
\hline$[16] /$ Experimental & 7500 & 1.14 & 0.411 & 0.203 \\
\hline$[16] /$ Numerical (LES) & 7500 & 1.23 & 0.359 & 0.200 \\
\hline$[17]$ / Numerical (SST) & 10000 & 1.57 & 1.20 & 0.235 \\
\hline Present / Numerical (SST) & 7500 & 1.59 & 1.19 & 0.258 \\
\hline Present / Experimental & 7500 & - & - & 0.200 \\
\hline
\end{tabular}

\section{Results and discussion}

All positions and lengths were normalized by diameter of the circular cylinder $(D)$ and all time-averaged velocity components and root-mean-square (RMS) values of the fluctuating velocity components were normalized by freestream velocity $\left(U_{\infty}\right)$. Figure 4 shows the averaged streamline topology on the measurement plane. Four cases are shown:

1) Bare cylinder, $B C$.

2) Cylinder with guiding plates located at $\alpha= \pm 70^{\circ}, \mathrm{A} 70$.

3) Cylinder with guiding plates located at $\alpha= \pm 100^{\circ}$, A100.

4) Cylinder with guiding plates located at $\alpha= \pm 130^{\circ}$, A130.

It is evident that there is a pair of recirculation cells in the near-wake of BC. It shows that vortex shedding from the BC occurs. For the case of A70, the size of these recirculation cells increased, i.e., vortex formation region is expanded. The size of the vortex formation region almost remained unchanged as angular positions of the plates from the front stagnation point of the cylinder increased from $\pm 70^{\circ}$ to $\pm 110^{\circ}$. In addition, a well-defined recirculation cell in the wake of the left plate as well as its counterpart on the opposite side of the wake centerline, are clearly apparent. It shows that vortex shedding from the guiding plates also begins to occur.

For the case of A100, two portion of the oncoming flow that entered the gaps between the cylinder and plates were directed slightly into the vortex formation region, whereas for the case of A130, they were penetrated the vortex formation region and substantially reduced the size of it. Guiding plates increased the streamwise velocity in the very near-wake (Fig. 5), i.e., reduced the pressure difference between the front and rear surfaces of the cylinder and presumably lead to a considerable reduction in the pressure (form) drag acting on the cylinder. On the other hand, at $\alpha= \pm 130^{\circ}$, one small recirculating cell in the wakes of the plates increased to two large recirculating cells, that is, pressure drag of the plates were increased.
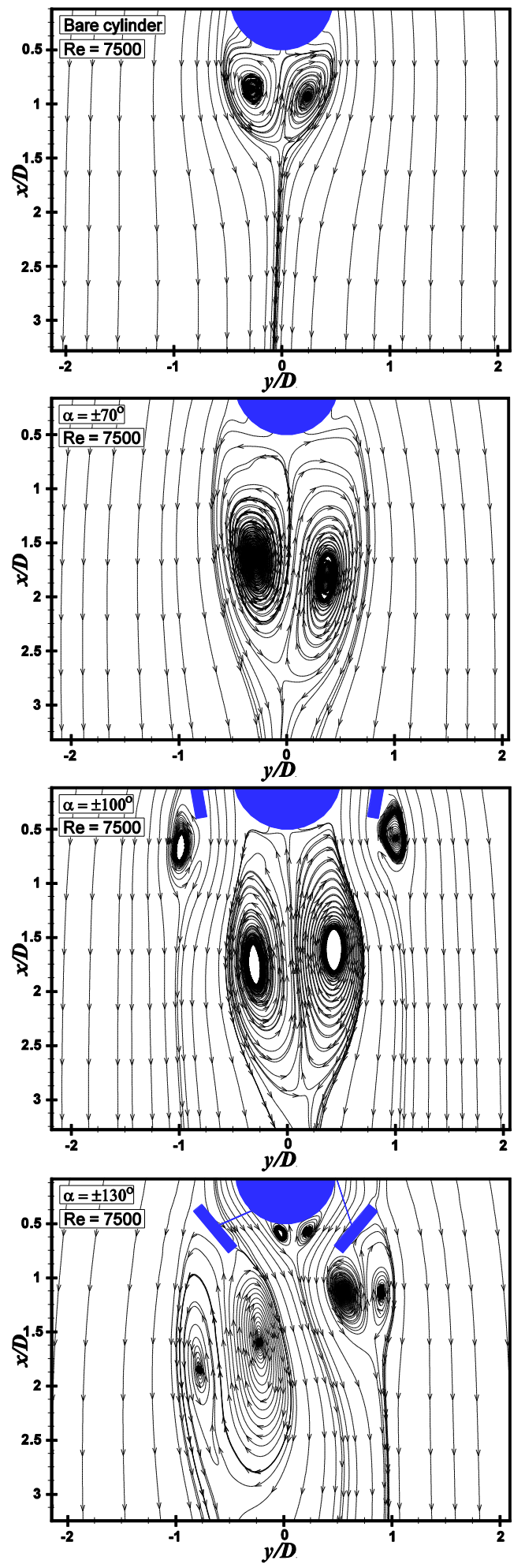

Fig. 4. Patterns of time-averaged streamline topology in the near-wake of the cylinder with and without the guiding plates. 


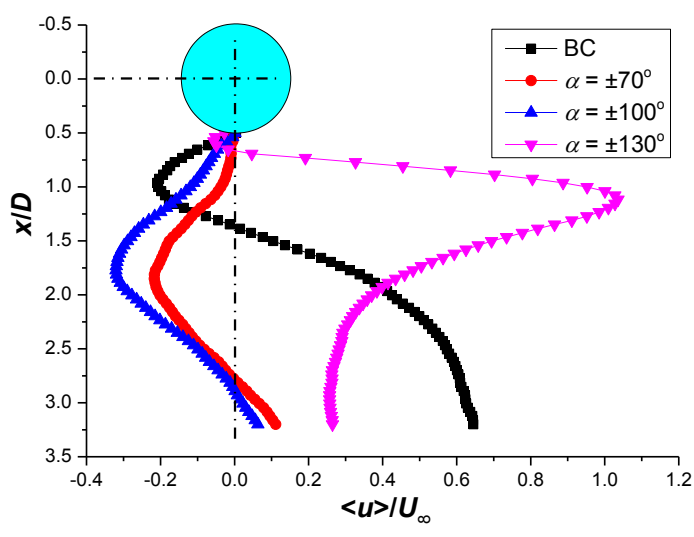

Fig. 5. Variation of $\langle u\rangle / U_{\infty}$ along the wake centerline for the BC, A70, A100 and A130 cases.

Figure 6 shows the patterns of averaged vorticity on the measurement plane. Low pressure region in the nearwake of the bare cylinder bent the shear layers from either sides of the cylinder towards the wake centerline. For the case of A70, the aforementioned shear layers were significantly extended in the streamwise direction. That is, vortex formation was displaced significantly downstream, relative to the case of $\mathrm{BC}$. This is also supported by the Fig. 7. Figure 7 shows that the vortex formation length (i.e. vertical distance between the center of the body and a point downstream of the body where the transverse velocity fluctuation level, $v_{r m s}$, has grown to a maximum) reaches a maximum in the A70 case. On the other hand, it reduced sharply with increased angle of the plate position simply because guiding plates started to obstruct the paths of the shear layers from the cylinder in the A100 case and greatly obstructed them in the A130 case. Hence, in A100 and A130 cases, shear layers from the cylinder bent towards the very near-wake of the cylinder. That is, in these cases, guiding plates are making the near-wake flow behaviour close to potential flow $(R e<1)$ patterns. Galvao et al. [7] stated that to make flow behavior closer to potential-flow patterns is a good guiding principle to eliminate vortex-induced vibration (VIV) while also reducing drag. Figure 7 also shows that the level of interaction between the two free shear layers from the cylinder is reduced by the existence of the guiding plates. That is, their strengths were reduced. Thus, the energy transferred from the fluid to the cylinder is reduced. As a result of this, VIV is reduced.

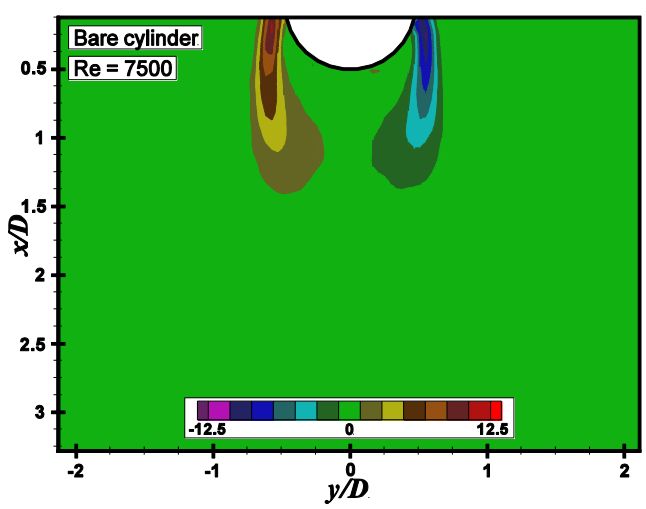

(Cont'd.)
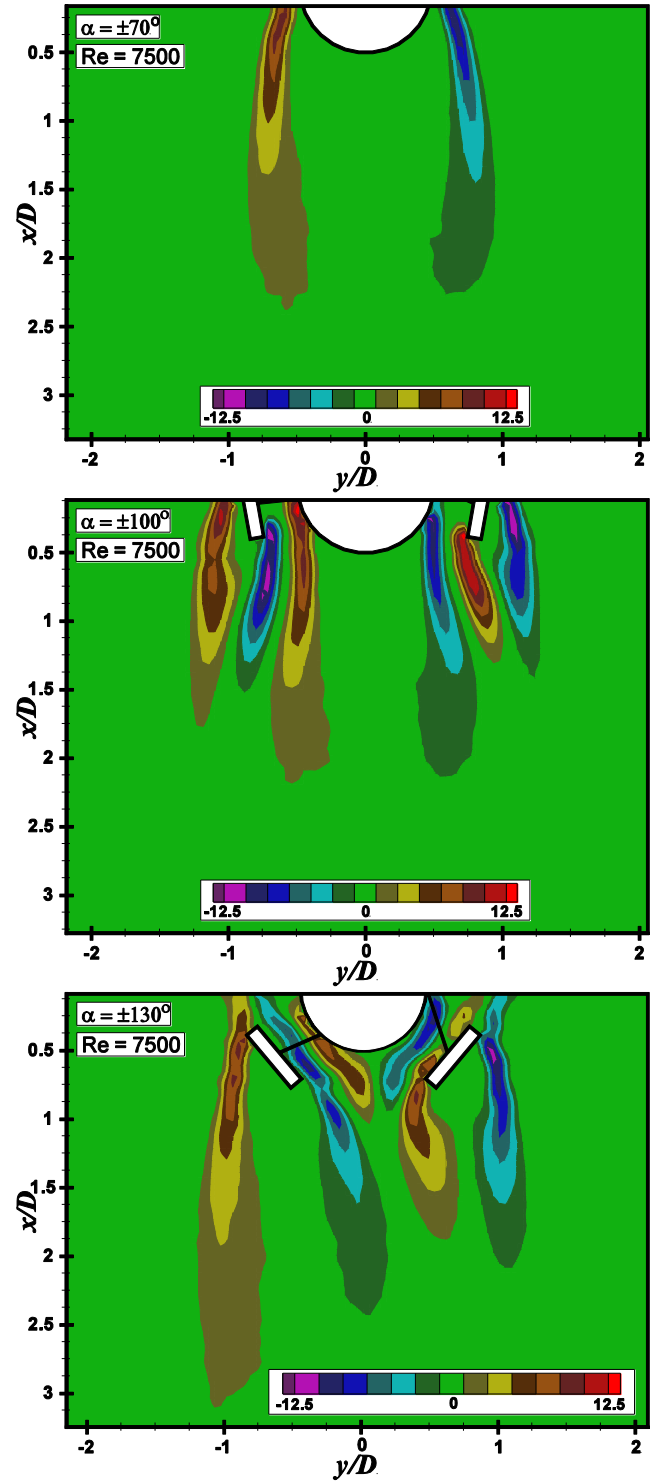

Fig. 6. Time-averaged patterns of spanwise vorticity in the near-wake of the cylinder with and without the guiding plates.

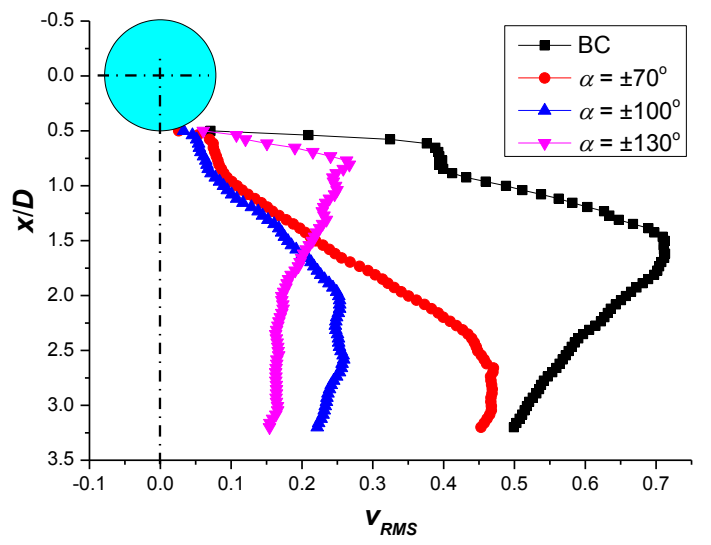

Fig. 7. Variation of $v_{\mathrm{rms}}$ along the wake centreline for the $\mathrm{BC}$, A70, A100 and A130 cases. 


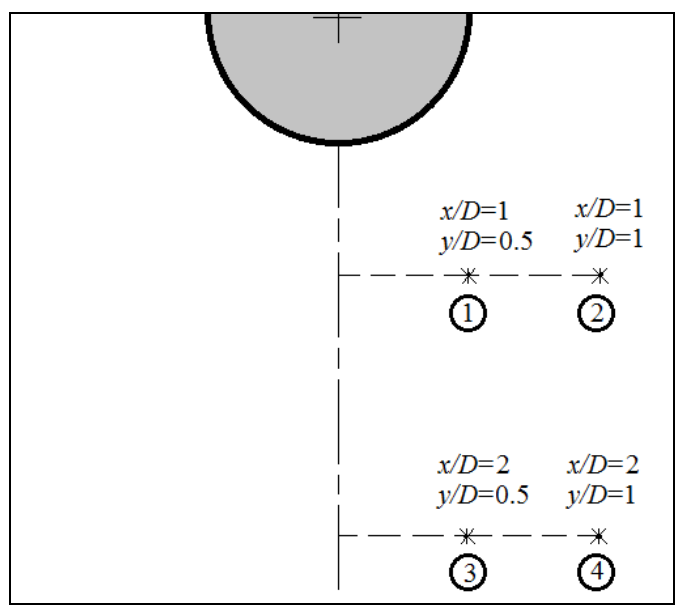

Fig. 8. The location and number of virtual transverse velocity probes behind the cylinder with and without the plates.
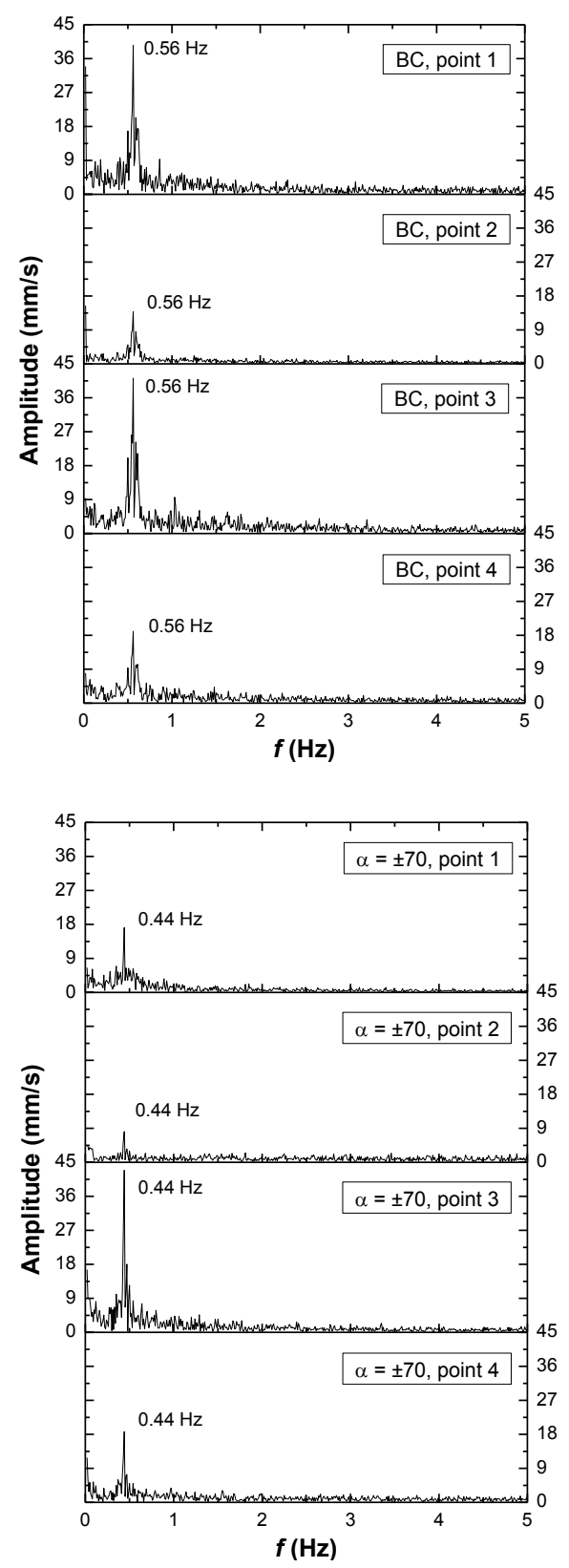

(Cont'd.)
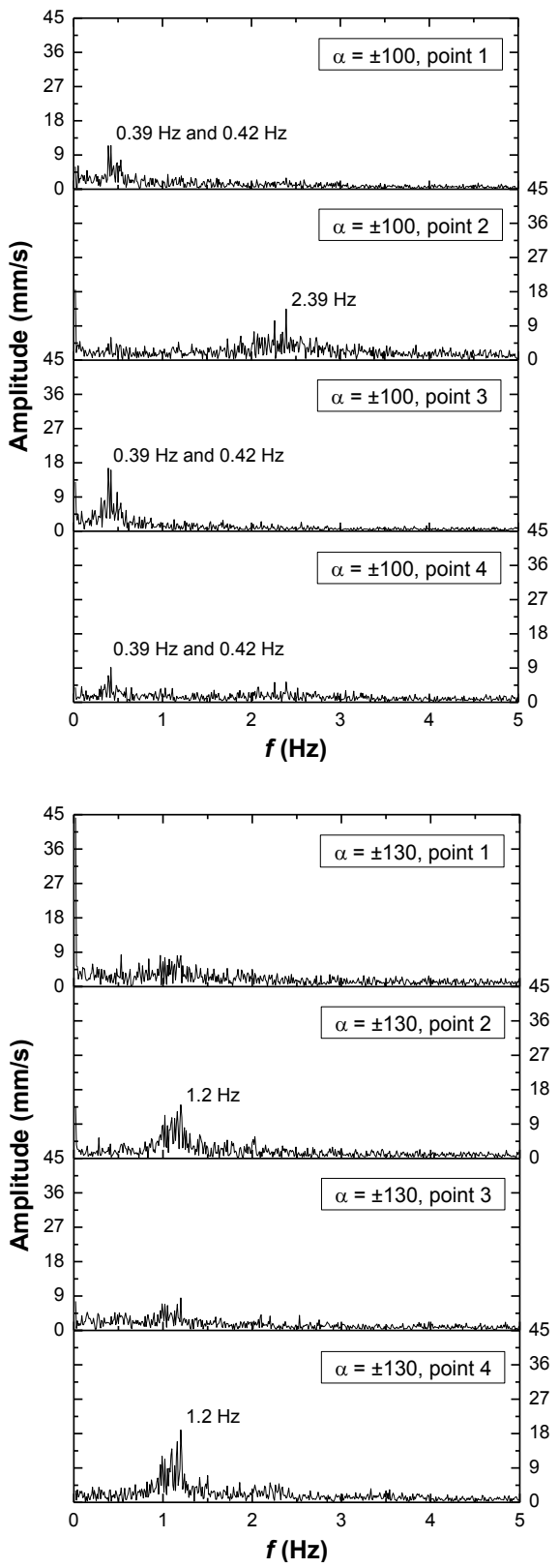

Fig. 9. Power spectra of the transverse velocity - time history at four locations.

A typical set of amplitude spectra of wake instantaneous transverse velocities at various positions through the near-wake (Fig. 8) is shown in Fig. 9. For the case of $\mathrm{BC}$, irrespective of the location of virtual probe, a well-defined spectral peak is observed at the vortex shedding frequency, $f=0.56 \mathrm{~Hz}$, corresponded to a Strouhal number $\left(S t=f \cdot D / U_{\infty}\right)$ of $S t=0.200$, and this is in agreement with value obtained by Norberg [12], 0.203.

The amplitude values of the spectral peaks at locations close to the wake centerline (i.e. points 1 and 3 ) are greater than those at locations far to the wake centerline (i.e. points 2 and 4 ) because their coordinates are inside the approximate boundary of vortex formation region where strong interaction occurs between the two shear layers from the cylinder. For the case of A70, the region of vortex formation was displaced noticeably downstream relative to the case of BC. Therefore, the amplitude value of the spectral peak at point 1 (Fig. 8) 
was reduced. Moreover, guiding plates at $\alpha= \pm 70^{\circ}$ noticeably retarded the onset and development of largescale vortical structures in the near-wake, relative to those occurring from a bare cylinder; thus, reduced the vortex shedding frequency from $0.56 \mathrm{~Hz}$ to $0.44 \mathrm{~Hz}$. For the cases of A100 and A130, irrespective of the location of the probe, there are no high peaks in the spectra. This means that guiding plates stabilized the near-wake by delaying the separation from the cylinder (Fig. 6) hence remove the tendency of the shear layers to react strongly with each other across the wake.

The force coefficients for the various cases considered are listed in table 2. Meanwhile, the frontal and planform area of the models are kept constant at $D \cdot 1$ $\mathrm{m}^{2}$ to compare directly the forces acting on models. The SST predictions suggest that guiding plates are not effective at drag reduction, but quite effective at vortex shedding suppression.

Table 2. Predicted time-averaged and rms force coefficients for cylinders with and without guiding plates.

\begin{tabular}{|l|c|c|}
\hline Cases simulated & $\boldsymbol{C}_{\boldsymbol{D}}$ & $\boldsymbol{C}_{\boldsymbol{L} \text { RMS }}$ \\
\hline Bare cylinder $(\mathrm{BC})$ & 1.59 & 1.19 \\
\hline A70 case $\left(\alpha= \pm 70^{\circ}\right)$ & 2.01 & 0.759 \\
\hline A100 case $\left(\alpha= \pm 100^{\circ}\right)$ & 1.58 & 0.538 \\
\hline A130 case $\left(\alpha= \pm 130^{\circ}\right)$ & 2.20 & 0.651 \\
\hline
\end{tabular}

\section{Conclusions}

The effects of guiding plates and their angular positions on the near-wake flow structure have been investigated experimentally and on the hydrodynamic coefficients have been predicted numerically. The results showed that guiding plates made a significant contribution to the reduction of the energy transfer from fluid to cylinder, that is, they are effective in suppression of vortexinduced vibrations (VIV) by extending the shear layers further downstream of the wake (A70) or by delaying the separation (A100 and A130). However, hydrodynamic coefficients predicted by two-dimensional unsteady RANS with SST $k$ - $\omega$ model indicate that guiding plates are not effective at drag reduction.

\section{References}

1. J.C. Owen, P.W. Bearman, J. Fluid Struct. 15, 597605 (2001)

2. H.-C. Lim, S.-J. Lee, AIAA J. 40, 2027-2036 (2002)

3. C.P. Shao, Q.D. Wei, AIAA J. 46, 397-407 (2008)

4. B. Zhou, X. Wang, W. Guo, J. Zheng, S.K. Tan, J Wind Eng. Ind. Aerodyn. 145, 30-41 (2015)

5. M. Sarioglu, Flow Meas. Instrum. 53, 221-229 (2017)

6. A.M. Hamed, J. Vega, B. Liu, L.P. Chamorro, Exp Fluids 58, 22 (2017)
7. R. Galvao, E. Lee, D. Farrell, F. Hover, M. Triantafyllou, N. Kitney, P. Beynet, J. Fluid Struct. 24, 1216-1226 (2008)

8. Y.E. Akansu, E. Firat, M. Hacialioğulları, Exp. Therm. Fluid Sci. 86, 11-22 (2017)

9. E. Firat, Y.E. Akansu, H. Akilli, Ocean Eng. 108, 504-518 (2015)

10. G.M. Ozkan, E. Firat, H. Akilli, Ocean Eng. 134, 35-49 (2017)

11. Engineering Science Data Unit, Data Item: ESDU 80025

12. C. Norberg, J. Fluid Struct. 17, 57-96 (2003)

13. F.R. Menter, AIAA J. 32, 1598-1605 (1994)

14. Fluent 6.3 User's Guide, 853-858 (2006)

15. A. Khalak, C.H.K. Williamson, J. Fluid Struct. 10, 455-472 (1996)

16. K. Lam, Y.F. Lin, L. Zou, Y. Liu, Int. J. Heat Fluid Flow 31, 32-44 (2010)

17. R.M. Stringer, J. Zang, A.J. Hills, Ocean Eng. 87, 19 (2014) 